\title{
ON THE ESTIMATION OF VON MISES EQUIVALENT STRESS IN RANDOM VIBRATION ANALYSIS
}

\author{
Federico Perotti, Francesco Foti and Luca Martinelli \\ Department of Civil and Environmental Engineering, Politecnico di Milano \\ Piazza Leonardo da Vinci, 3220133 Milan Italy \\ \{federico.perotti, francesco.foti, luca.martinelli\}@polimi.it
}

Keywords: structural dynamics, random vibration, seismic analysis, stress assessment

\begin{abstract}
Linear random vibration analysis aims to the probabilistic characterization of response functions; classical procedures are targeted to the time histories of output kinematic variables, such as displacement, velocity and acceleration, and to parameters which are linearly related to them, such as internal forces, deformations, stress components ecc. Much less attention has been devoted, by researcher and developers, to the case of response variables which are non-linearly dependent of the lagrangian coordinates or their derivatives; the most typical among these is the Von Mises equivalent stress, whose square value is quadratic in the components of the stress tensor, and thus in the lagrangian coordinates. The topic is of relevance especially for the structural safety of industrial equipment and systems, which is usually based on local stress integrity assessment.

In the paper an innovative analytical procedure to deal with the probabilistic characterization of the VM stress is proposed as an extension of the classical approach adopted for linear output parameters. To this aim the matrix of the quadratic form delivering is decomposed into the sum of suitable factors; each of them deserves the same treatment as for linear parameters.

The various features related to the numerical implementation of the procedure for stationary or non-stationary (evolutionary) random excitation are discussed, with reference to both the application via direct frequency domain treatment and to the modal superposition approach; an example is finally shown and commented.
\end{abstract}




\section{INTRODUCTION}

Linear random vibration analysis is a powerful tool for the probabilistic characterization of the response of linearized systems to dynamic perturbative forces, both for the case of environmental interactions and for excitations of anthropic nature.

Aim of the analysis is usually the assessment of both the functionality under serviceability loads and the structural integrity under exceptional loads. The first is naturally checked in the linear range, in which no damage occurs. For the second, which should imply inelastic behavior and/or large deformations, elastic methods are nevertheless accepted in many situations. A typical example is represented by the integrity assessment of equipment components in industrial plants, which can be performed (see ASME B31.30) by means of elastic methods against complex damage phenomena, such as ratcheting, ....

Linear random vibration analysis aims to the probabilistic characterization of response functions; classical procedures are targeted to the time histories of output kinematic variables, such as displacement, velocity and acceleration, and to parameters which are linearly related to them, such as internal forces, deformations, stress components ecc. Much less attention has been devoted to the case of response variables which are non-linearly dependent of the lagrangian coordinates or their derivatives; the most typical among these is the Von Mises equivalent stress, whose square value is quadratic in the components of the stress tensor, and thus in the lagrangian coordinates. The topic is crucial for the structural safety of industrial equipment and systems, which is usually based on local stress integrity assessment.

The extreme-value distribution of the Von Mises (VM in the following) stress is addressed in [1] by estimating, through the Rice formula, the outcrossing rate of an ellipsoid by the stress component vector process; the derivation is performed for stationary gaussian random processes. In the same context, in [2] the estimation of the RMS value of the VM stress is pursued; classical approaches are here exploited such as modal analysis and frequency domain integration.

In this paper the approach proposed in [2] is cast into the more general framework of nonstationary (evolutionary) random processes. For the stationary case an innovative analytical procedure to deal with the probabilistic characterization of the VM stress is proposed as an extension of the classical approach adopted for linear output parameters. To this aim the matrix of the quadratic form delivering the equivalent stress is decomposed into the sum of suitable factors; each of them deserves the same treatment as for linear parameters. The various features related to the numerical implementation of the procedure for stationary or nonstationary (evolutionary) random excitation are discussed, with reference to both the application via direct frequency domain treatment and to the modal superposition approach; an example is finally shown and commented.

\section{STRESS RESPONSE TO RANDOM DYNAMIC EXCITATION}

The response of a linear $n$ dof system is considered, governed by the following equations of motion

$$
\mathbf{m} \ddot{\mathbf{q}}(t)+\mathbf{c} \dot{\mathbf{q}}(t)+\mathbf{k q}(t)=\mathbf{F} \mathbf{g}(t)
$$

where $\mathbf{m}, \mathbf{c}$ and $\mathbf{k}$ are respectively the mass, damping and stiffness matrices, $\mathbf{F}$ is a matrix of load amplitude coefficients and $\mathbf{g}(t)$ is the vector listing the functions that define the time variation of the loads.

Assuming non-stationary (evolutionary) random excitation, the time histories of the loads can be expressed (see Priestley [3]) according to the Fourier-Stieljes integral formulation 


$$
\mathbf{g}(t)=\int_{-\infty}^{\infty} \boldsymbol{\Psi}(t, f) \exp (i 2 \pi f t) d \mathbf{G}(f)
$$

where $\Psi(t, f)$ is a diagonal matrix listing deterministic, slowly varying envelope functions. $d \mathbf{G}(f)$ is an orthogonal process related to a zero-mean stationary process, having a Spectral Power Density (SPD) $\mathbf{S}_{g}(f)$, through the relation

$$
E\left[d \mathbf{G}\left(f_{1}\right) d \mathbf{G}^{*}\left(f_{2}\right)\right]=\delta\left(f_{1}-f_{2}\right) \mathbf{S}_{g}\left(f_{1}\right)
$$

In such setting, the response of the system can be expressed, by integrating in the frequency domain, through the following expression

$$
\mathbf{q}(t)=\int_{-\infty}^{\infty} \boldsymbol{\Gamma}(t, f) \exp (i 2 \pi f t) d \mathbf{G}(f)
$$

The $\boldsymbol{\Gamma}(t, f)$ function appearing in (4) is in turn obtained via the time convolution

$$
\boldsymbol{\Gamma}(t, f)=\int_{-\infty}^{\infty} \mathbf{h}(u) \mathbf{F} \boldsymbol{\Psi}(t-u, f) \exp (-2 \pi f u) d u
$$

where $\mathbf{h}(u)$ is the matrix of the unit impulse response functions of the system.

If we now express a generic stress component as a linear combination of the configuration vector components we can write:

$$
\sigma_{j k}(t)=\mathbf{z}^{T} \mathbf{q}(t)=\mathbf{z}^{T} \int_{-\infty}^{\infty} \boldsymbol{\Gamma}(t, f) \exp (i 2 \pi f t) d \mathbf{G}(f)
$$

so that

$$
\sigma_{j k}^{2}(t)=\mathbf{q}^{T}(t) \mathbf{z} \mathbf{z}^{T} \mathbf{q}(t)=\mathbf{z}^{T} \mathbf{q}(t) \mathbf{q}^{T}(t) \mathbf{z}
$$

Note that, if modal analysis is performed, by expressing the configuration vector as a linear combination $\mathbf{q}(t)=\boldsymbol{\Phi} \mathbf{y}(t)$ of the system eigenvectors, we get the following

$$
\sigma_{j k}^{2}(t)=\mathbf{q}^{T}(t) \mathbf{z} \mathbf{z}^{T} \mathbf{q}(t)=\mathbf{y}^{T}(t) \mathbf{u} \mathbf{u}^{T} \mathbf{y}(t)
$$

where $\mathbf{u}=\mathbf{\Phi} \mathbf{z}$ and $\mathbf{y}(t)$ is the vector of modal coordinates.

If direct analysis is performed, i.e. without modal expansion, the mean square value of the stress component can thus be expressed, from (7), as

$$
E\left[\sigma_{j k}^{2}(t)\right]=\mathbf{z}^{T} E\left[\mathbf{q}(t) \mathbf{q}^{T}(t)\right] \mathbf{z}=\mathbf{z}^{T} \boldsymbol{\mu}_{q} \mathbf{z}
$$

i.e. as a function of the covariance matrix $\boldsymbol{\mu}_{q}$; taking expressions (3) to (5) into account the latter can be in turn obtained from the following derivation

$$
\begin{aligned}
\boldsymbol{\mu}_{q} & =E\left[\mathbf{q}\left(f_{1}\right) \mathbf{q}^{T}\left(f_{2}\right)\right]= \\
& =\left[\int_{-\infty}^{\infty} \int_{-\infty}^{\infty} \boldsymbol{\Gamma}\left(t, f_{1}\right) E\left[d \mathbf{G}\left(f_{1}\right) d \mathbf{G}^{*}\left(f_{2}\right)\right] \boldsymbol{\Gamma}^{*}\left(t, f_{2}\right) \exp \left(i 2 \pi\left(f_{1}-f_{2}\right) t\right)\right]= \\
& =\left[\int_{-\infty}^{\infty} \int_{-\infty}^{\infty} \boldsymbol{\Gamma}\left(t, f_{1}\right) \mathbf{S}_{g}\left(f_{1}\right) \boldsymbol{\Gamma}^{*}\left(t, f_{2}\right) \delta\left(f_{1}-f_{2}\right) \exp \left(i 2 \pi\left(f_{1}-f_{2}\right) t\right) d f_{1} d f_{2}\right]= \\
& =\int_{-\infty}^{\infty} \boldsymbol{\Gamma}(t, f) \mathbf{S}_{g}(f) \boldsymbol{\Gamma}^{*}(t, f) d f
\end{aligned}
$$


A time-varying (evolutionary) SPD is consistently defined as:

$$
S_{\sigma_{j k}}(f, t)=\mathbf{z}^{T} \boldsymbol{\Gamma}(t, f) \mathbf{S}_{g}(f) \boldsymbol{\Gamma}^{*}(t, f) \mathbf{z}=\mathbf{z}^{T} \mathbf{S}_{q}(f, t) \mathbf{z}
$$

For a stationary excitation we get the following:

$$
\mathbf{\Psi}(f, t)=\mathbf{I} \quad ; \quad \boldsymbol{\Gamma}(f, t)=\mathbf{H}(f) \mathbf{F} \quad ; \quad S_{\sigma_{j k}}(f)=\mathbf{z}^{T} \mathbf{H}(f) \mathbf{F} \mathbf{S}_{g}(f) \mathbf{F}^{T} \mathbf{H}^{*}(f) \mathbf{z}
$$

in which $\mathbf{H}(f)$ is the usual matrix of Frequency Response Functions, i.e. the inverse of the Mechanical Impedance matrix $\mathbf{E}(f)$, so that

$$
\mathbf{H}(f)=\mathbf{E}^{-1}(f)=\left[-(2 \pi f)^{2} \mathbf{m}+(i 2 \pi f) \mathbf{c}+\mathbf{k}\right]^{-1}
$$

\section{THE ESTIMATION OF THE VON MISES EQUIVALENT STRESS}

Starting from the stress tensor components the equivalent Von Mises stress can be expressed by the following quadratic form

$$
\sigma_{V M}^{2}(t)=\boldsymbol{\sigma}^{T}(t) \mathbf{A} \boldsymbol{\sigma}(t)
$$

where $\mathbf{A}$ is a $6 x 6$ symmetric positive definite matrix, whose entries are given in the Appendix. By expressing the stress components as linear functions of the configuration we get

$$
\sigma_{V M}^{2}(t)=\boldsymbol{\sigma}^{T}(t) \mathbf{A} \boldsymbol{\sigma}(t)=\mathbf{q}^{T}(t) \mathbf{Z} \mathbf{A} \mathbf{Z}^{T} \mathbf{q}(t)=\mathbf{q}^{T}(t) \mathbf{B} \mathbf{q}(t)
$$

in which $\boldsymbol{Z}$ is a matrix of dimension $6 x n_{c}$, being $n_{c}$ the number of lagrangian coordinates affecting the computation of the stress tensor $\sigma$.

Expressions (15), (4) and (5) formally solve the problem of the Von Mises stress evaluation. When the evaluation of the expected value of the squared VM stress is addressed, however, it can be easily verified that an approach similar to equations (8)-(10) is problematic. To overcome these problems, the matrix $\mathbf{B}$, symmetric and positive definite, can be decomposed in terms of its eigenvectors, i.e.

$$
\begin{gathered}
\mathbf{B} \overline{\mathbf{R}}=\overline{\mathbf{R}} \boldsymbol{\Lambda} \rightarrow \mathbf{B}=\overline{\mathbf{R}} \Lambda^{1 / 2} \boldsymbol{\Lambda}^{1 / 2} \overline{\mathbf{R}}^{T}=\mathbf{R} \mathbf{R}^{T} \\
\sigma_{V M}^{2}(t)=\mathbf{q}^{T}(t) \mathbf{R} \mathbf{R}^{T} \mathbf{y}(t)=\mathbf{q}^{T}(t)\left(\sum_{i=1}^{M} \mathbf{r}_{i} \mathbf{r}_{i}^{T}\right) \mathbf{q}(t)=\sum_{i=1}^{M} \mathbf{r}_{i}^{T} \mathbf{q}(t) \mathbf{q}^{T}(t) \mathbf{r}_{i}
\end{gathered}
$$

It is worth noting that, having applied the eigenvector transformation, we have obtained in (17) an expression which is simply the sum of $M$ terms having exactly the same form as obtained in (8) for the linear response parameter (generic stress component); one the expectation operator is applied, it can be now interchanged with the summation, leading to an expression which is the sum of terms sharing the same form as in (9), i.e.

$$
E\left[\sigma_{V M}^{2}(t)\right]=E\left[\sum_{i=1}^{M} \mathbf{r}_{i}^{T} \mathbf{q}(t) \mathbf{q}^{T}(t) \mathbf{r}_{i}\right]=\sum_{i=1}^{m} \mathbf{r}_{i}^{T} E\left[\mathbf{q}(t) \mathbf{q}^{T}\right] \mathbf{r}_{i}=\sum_{i=1}^{m} \mathbf{r}_{i}^{T} \boldsymbol{\mu}_{q} \mathbf{r}_{i}
$$

The efficiency of the proposed procedure deserves further comments when applied within the context of the modal superposition approach. In such setting, by expressing the configuration vector as a linear combination $\mathbf{q}(t)=\boldsymbol{\Phi} \mathbf{y}(t)$ of the system eigenvectors, we get the following alternative formulation

$$
\begin{gathered}
\sigma_{V M}^{2}(t)=\boldsymbol{\sigma}^{T}(t) \mathbf{A} \boldsymbol{\sigma}(t)=\mathbf{q}^{T}(t) \mathbf{Z A} \mathbf{A} \mathbf{Z}^{T} \mathbf{q}(t)=\mathbf{y}^{T}(t) \boldsymbol{\Phi}^{T} \mathbf{Z} \mathbf{A} \mathbf{Z}^{T} \boldsymbol{\Phi} \mathbf{y}(t) \\
=\mathbf{y}^{T}(t) \boldsymbol{\beta}^{T} \mathbf{A} \boldsymbol{\beta} \mathbf{y}(t)=\mathbf{y}^{T}(t) \mathbf{D y}(t)
\end{gathered}
$$


It can be observed, on practical grounds, that each row of the $(n, 6) \boldsymbol{\Phi}^{T} \mathbf{Z}$ matrix lists the components of the stress tensor occurring when the corresponding modal shape is imposed to the system; these are often available through commercial software, so that the final matrix $\mathbf{D}$ can be assumed as available also for large and complex structural models.

Again, the eigenvalue decomposition of the matrix $\mathbf{D}$, which is symmetric and positive definite, can be considered, leading to the following factorization:

$$
\mathbf{D} \overline{\mathbf{S}}=\overline{\mathbf{S}} \boldsymbol{\Lambda} \rightarrow \mathbf{D}=\overline{\mathbf{S}} \boldsymbol{\Lambda}^{1 / 2} \boldsymbol{\Lambda}^{1 / 2} \overline{\mathbf{S}}^{T}=\mathbf{S} \mathbf{S}^{T}
$$

Since $\mathbf{D}$, from its definition (19), has maximum rank equal to six, the squared VM stress can be now expressed as a quadratic form in the normal coordinates given as the combination of six terms having the same form as in equation (8), i.e.

$$
\begin{aligned}
\sigma_{V M}^{2}(t)=\mathbf{y}^{T}(t) \mathbf{S S}^{T} \mathbf{y}(t) & =\mathbf{y}^{T}(t)\left(\sum_{i=1}^{M} \mathbf{s}_{i} \mathbf{s}_{i}^{T}\right) \mathbf{y}(t)=\sum_{i=1}^{M} \mathbf{y}^{T}(t) \mathbf{s}_{i} \mathbf{s}_{i}^{T} \mathbf{y}(t)= \\
& =\sum_{i=1}^{M} \mathbf{s}_{i}^{T} \mathbf{y}(t) \mathbf{y}^{T}(t) \mathbf{s}_{i}
\end{aligned}
$$

The computation of (19) requires availability of the $(p, M)$ matrix $\mathbf{S}$ (with $M \leq 6$ ), which can be clearly much less expensive than the direct storage of $\mathbf{D}$, the latter being implicit in the method proposed in [2].

The variance of the VM stress can thus be expressed in terms of the covariances $\sigma_{y_{j} y_{k}}$ among normal-mode response functions, i.e.

$$
E\left[\sigma_{V M}^{2}(t)\right]=\sum_{i=1}^{M} \mathbf{s}_{i}^{T} E\left[\mathbf{y}(t) \mathbf{y}^{T}(t)\right] \mathbf{s}_{i}=\sum_{i=1}^{M} \sum_{j=1}^{p} \sum_{k=1}^{p} s_{i, j} \sigma_{y_{j} y_{k}} s_{i, k}
$$

In the stationary case $\sigma_{y_{j} y_{k}}$ can be, in turn, expressed via the following frequency integral

$$
\sigma_{y_{j} y_{k}}=\int_{-\infty}^{+\infty} S_{y_{j} y_{k}}(f) d f=\boldsymbol{\varphi}_{j}^{T} \mathbf{F} \int_{-\infty}^{+\infty} \bar{H}_{j}(f) \bar{H}_{k}(f) \mathbf{S}_{g}(f) d f \mathbf{F}^{T} \boldsymbol{\varphi}_{k}
$$

In the integral (21) the modal Frequency Response Functions (FRFs) appear, defined as:

$$
\bar{H}_{j}(f)=\frac{1}{\left(2 \pi f_{j}\right)^{2} M_{j}} \frac{1}{1-\frac{f^{2}}{f_{j}^{2}}+i 2 \xi_{j} \frac{f}{f_{j}}}=\frac{1}{\left(2 \pi f_{j}\right)^{2} M_{j}} \tilde{H}_{j}(f)
$$

In (22) $f_{j}, M_{j}$ and $\xi_{j}$ are respectively the modal frequency, mass and damping ratio.

In the case of perfect correlation of input forces, typical of one-component seismic excitation, the input spectral matrix becomes a scalar SPD; in addition, in can be noted that:

$$
\boldsymbol{\varphi}_{j}^{T} \mathbf{F}=-\boldsymbol{\varphi}_{j}^{T} \mathbf{m r}=-\Gamma_{j} M_{j}
$$

where $\mathbf{r}$ is a vector having non zero (unit) components along coordinates expressing displacement components parallel to the seismic input and $\Gamma_{j}$ is the usual modal participation factor. Upon substitution of $(24,25)$ into $(23)$ we obtain the following:

$$
\sigma_{y_{j} y_{k}}=\int_{-\infty}^{+\infty} S_{y_{j} y_{k}}(f) d f=\frac{\Gamma_{j} \Gamma_{k}}{16 \pi^{4} f_{j}^{2} f_{k}^{2}} \int_{-\infty}^{+\infty} \tilde{H}_{j}(f) \tilde{H}_{k}(f) S_{u ̈}(f) d f
$$

in which the SPD of the free-field ground acceleration is now introduced in the integral. 
It is worth noting that all the quantities appearing in equation (26) can be easily computed (the integral) or obtained by standard structural analysis software, as the participation factors and modal frequencies. Given that the matrix $\mathbf{D}$ can be obtained as well from the standard code, and its eigenvalues easily computed, the evaluation of the mean square VM stress is easily at hand.

Finally, the most general case in which 3D seismic excitation is applied in addition to static loading can be addressed upon simple superposition of effects, by stating:

$$
\sigma_{V M}^{2}(t)=\left\{\boldsymbol{\sigma}^{(x) T}(t)+\boldsymbol{\sigma}^{(y) T}(t)+\boldsymbol{\sigma}^{(z) T}(t)+\boldsymbol{\sigma}^{(s t) T}\right\} \mathbf{A}\left\{\boldsymbol{\sigma}^{(x)}(t)+\boldsymbol{\sigma}^{(y)}(t)+\boldsymbol{\sigma}^{(z)}(t)+\boldsymbol{\sigma}^{(s t)}\right\}
$$

By expressing the stress vector in terms of the normal response, applying again the eigenvalue decomposition to the matrix $\mathbf{D}$, considering that the normal coordinates are zero-mean processes and taking the average of (27) it can be derived that the SRSS rule can be adopted for combining the static effect and the effect of the three components of excitation, provided that the latter are uncorrelated.

\section{CASE STUDY}

The preceding criteria have been applied, as a first test, to a problem obtained upon drastic simplification of a real life case; this was an equipment component located in a seismically isolated industrial building. Isolation was acting only in the horizontal plane; accordingly, the vertical excitation applied to the component is by far the most significant.
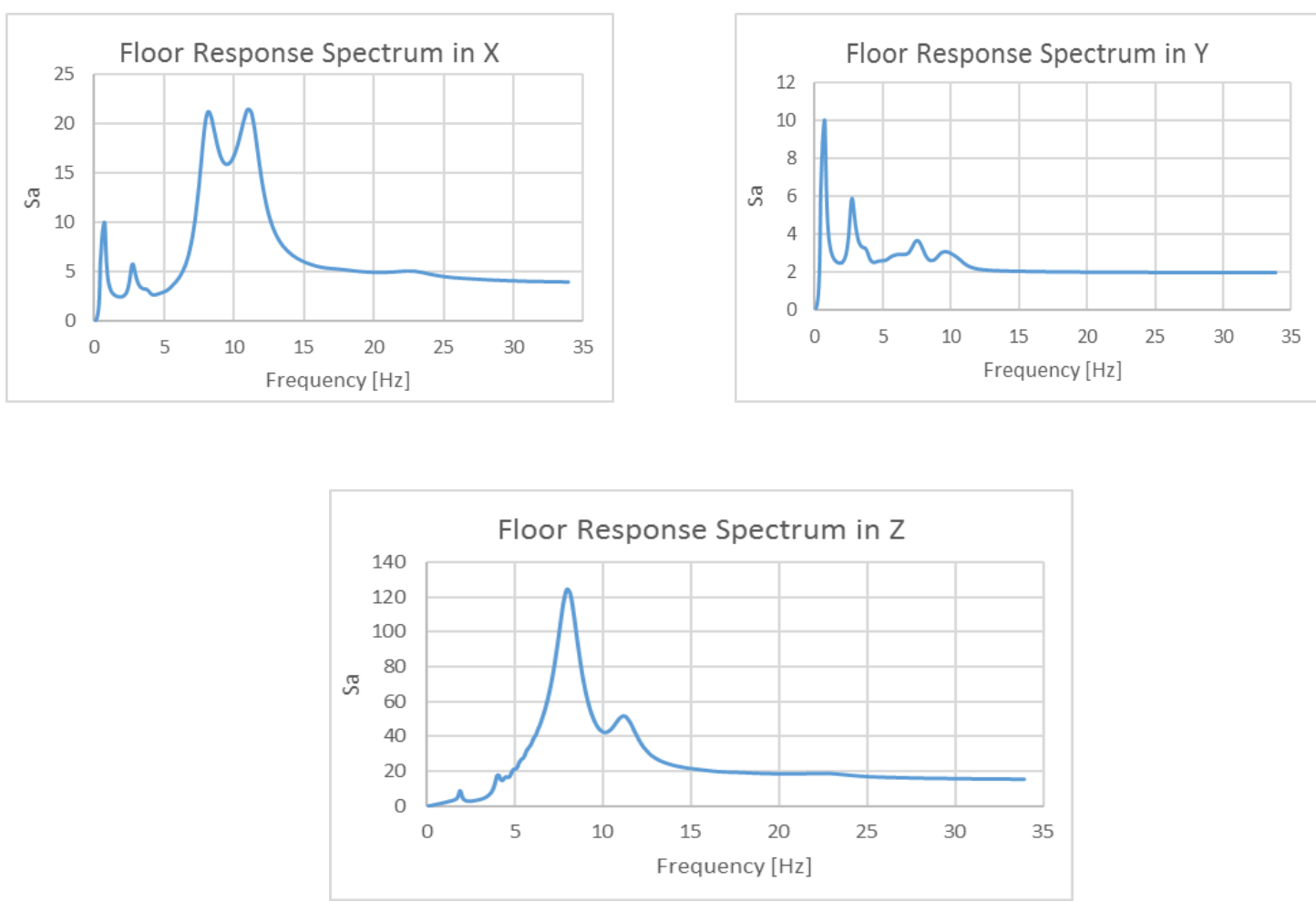

Figure1. Floor response spectrum in X, Y (horizontal) and Z (vertical) direction.

Figures 1 and 2 show the floor response spectra (FRS) applied to the system and a triplet of spectrum-compatible accelerograms obtained from the FRS; seven triplets were generated for the subsequent comparisons. In Table 1 the natural frequencies of the first ten normal modes of the model are given, spanning a range which is quite typical for a broad class of equipment components. 

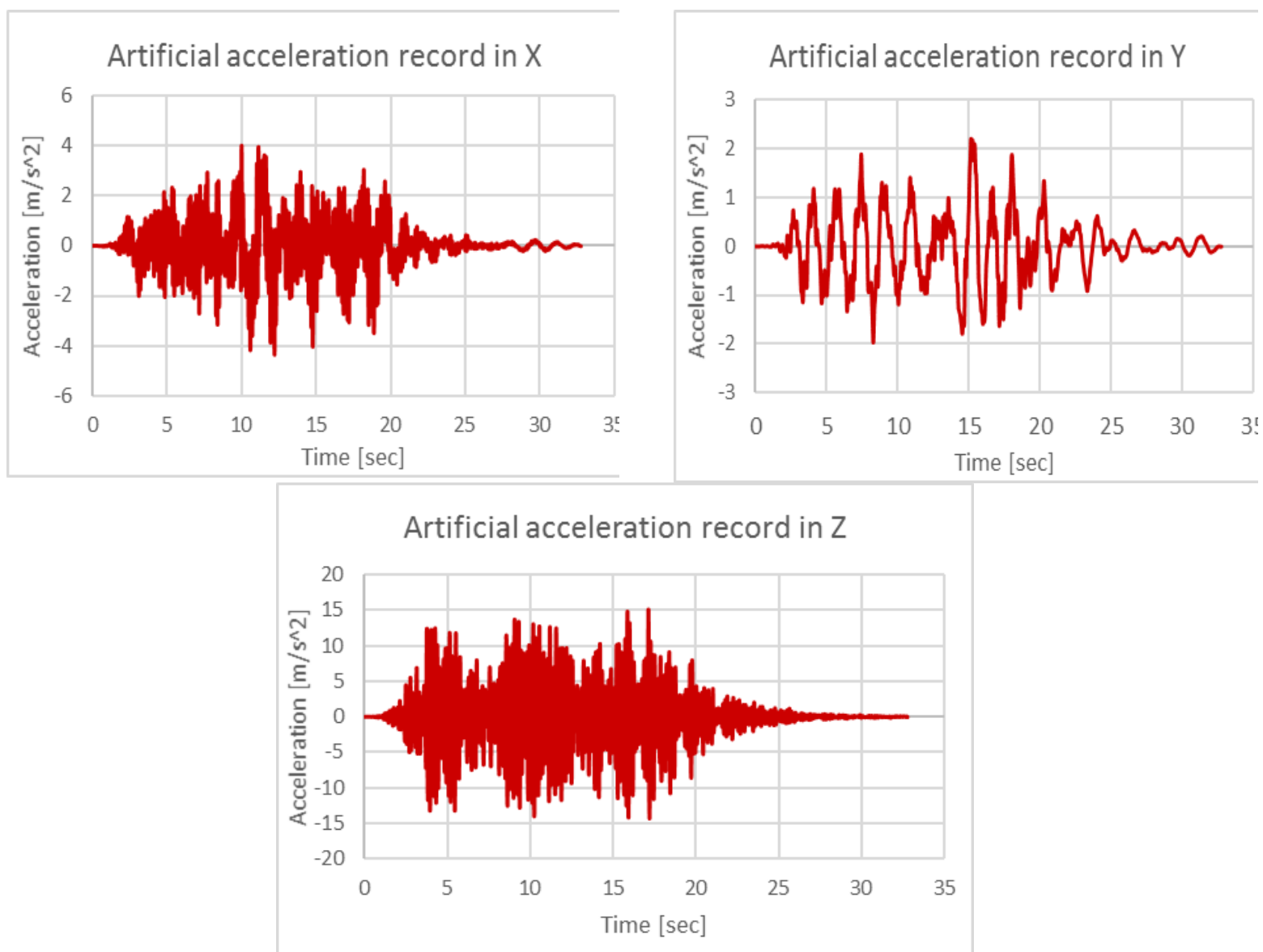

Figure 2. Artificial acceleration records in X, Y (horizontal) and Z (vertical) direction.

\begin{tabular}{|l|l|l|l|l|}
\hline 11.728 & 11.755 & 40.698 & 61.899 & 85.527 \\
\hline 127.03 & 171.51 & 202.23 & 207.04 & 284.71 \\
\hline
\end{tabular}

Table 1: Natural frequencies [Hz] of the example model.

The subsequent figure 3 shows the Finite Element mesh and the point where the stress assessment was performed. In these points the mean square Von Mises stress has been evaluated according to the following procedures:

- application of formulas (22) and (23),

- step-by-step time domain analysis, performed for the seven FRS compatible triplets of accelerograms; the mean square VM stress is obtained by simply averaging the values obtained in the seven cases,

- application of the ANSYS procedure based on [2].

In the following tables the result of the comparison is shown, suggesting the following general comments;

- the proposed method outcomes are generally in excellent agreement with the ones of the ANSYS procedure,

- both random vibration based procedures deliver slightly larger results when compared to step-by-step analysis, this being justified by the stationarity assumption. 

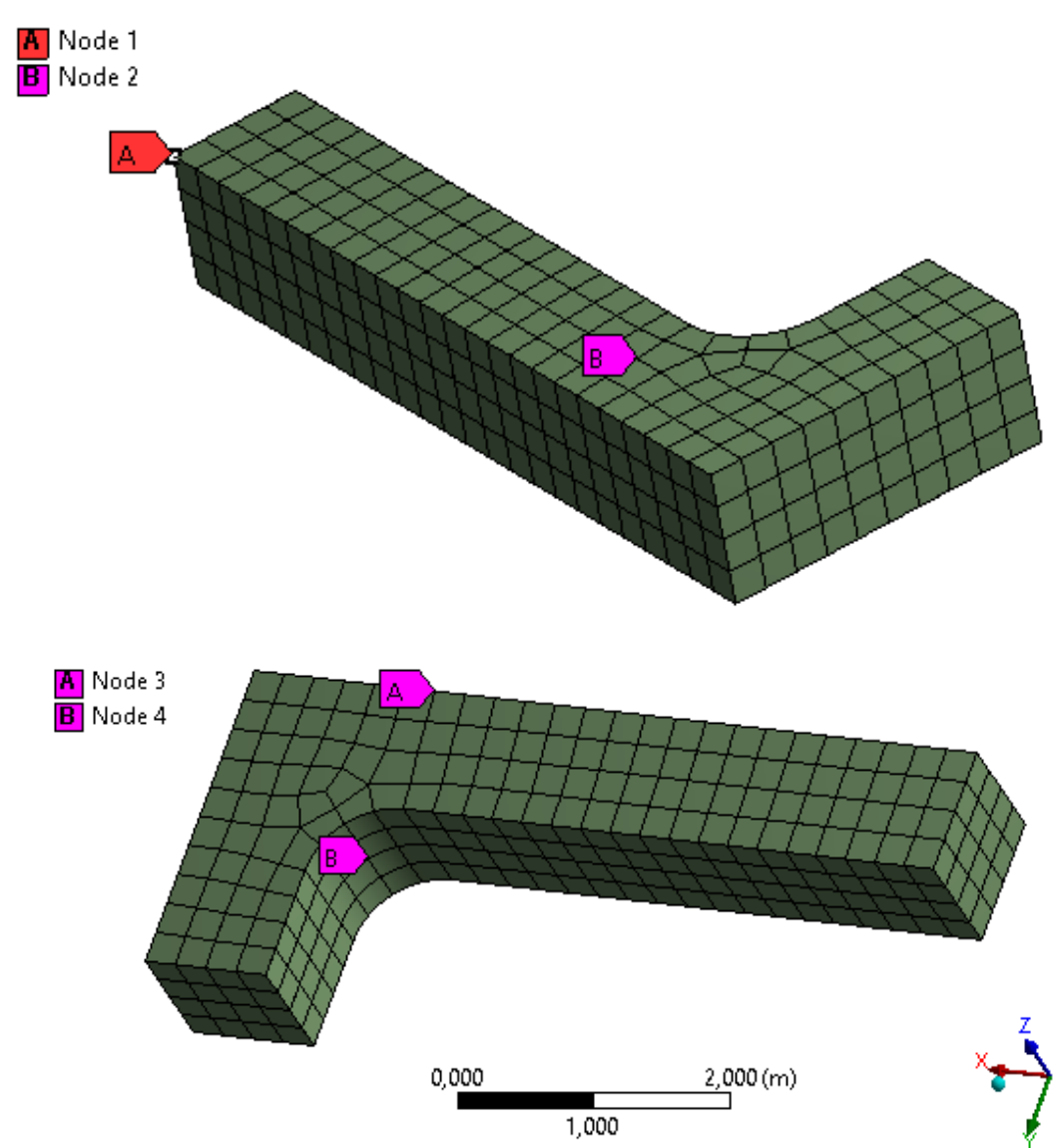

Figure 3. FE mesh and points for stress evaluation.

\begin{tabular}{|c|c|c|c|c|c|}
\hline \multirow{2}{*}{ Direction } & T History & RV Ansys & $\begin{array}{c}\text { RV } \\
(22-23)\end{array}$ & $\begin{array}{c}\text { Error TH } \\
\text { vs. Ansys }\end{array}$ & $\begin{array}{c}\text { Error TH } \\
\text { vs. RV }\end{array}$ \\
\cline { 2 - 6 } & {$[\mathrm{MPa}]$} & {$[\mathrm{MPa}]$} & {$[\mathrm{MPa}]$} & {$[\%]$} & {$[\%]$} \\
\hline $\mathrm{X}$ & 1,20 & 1,29 & 1,29 & 7,65 & 8,13 \\
\hline $\mathrm{Y}$ & 1,82 & 2,18 & 2,08 & 19,75 & 14,50 \\
\hline $\mathrm{Z}$ & 27,82 & 30,06 & 30,22 & 8,05 & 8,63 \\
\hline
\end{tabular}

Table 2: stress evaluation at point 1

\section{CONCLUSIONS}

A numerical procedure has been proposed in the paper for estimating the RMS value of the Von Mises equivalent stress within a random vibration analysis. The procedure is based on a transformation allowing the VM stress, which is a quadratic form in the coordinates, to be represented as the sum of linear output quantities; on this basis standard procedures targeted to the latter parameters can be exploited.

Some tests performed on a simple FE system are shown, in which the results obtained by means of the procedure are satisfactorily compared to the ones obtained by the method available in the software ANSYS and to the ones computed by a time-domain step-by-step procedure. The results here shown open the way to applications performed via more common design approaches, such as the response spectrum method. 


\begin{tabular}{|c|c|c|c|c|c|}
\hline \multirow{2}{*}{ Direction } & T History & RV Ansys & $\begin{array}{c}\text { RV } \\
(22-23)\end{array}$ & $\begin{array}{c}\text { Error TH } \\
\text { vs. Ansys }\end{array}$ & $\begin{array}{c}\text { Error TH } \\
\text { vs. RV }\end{array}$ \\
\cline { 2 - 6 } & {$[\mathrm{Mpa}]$} & {$[\mathrm{Mpa}]$} & {$[\mathrm{Mpa}]$} & {$[\%]$} & {$[\%]$} \\
\hline $\mathrm{X}$ & 0,12 & 0,13 & 0,13 & 8,02 & 8,38 \\
\hline $\mathrm{Y}$ & 0,12 & 0,20 & 0,13 & 72,42 & 14,26 \\
\hline $\mathrm{Z}$ & 4,34 & 4,90 & 4,92 & 12,99 & 13,53 \\
\hline
\end{tabular}

Table 3: stress evaluation at point 2

\begin{tabular}{|c|c|c|c|c|c|}
\hline \multirow{2}{*}{ Direction } & T History & RV Ansys & $\begin{array}{c}\text { RV } \\
(22-23)\end{array}$ & $\begin{array}{c}\text { Error TH } \\
\text { vs. Ansys }\end{array}$ & $\begin{array}{c}\text { Error TH } \\
\text { vs. RV }\end{array}$ \\
\cline { 2 - 6 } & {$[\mathrm{Mpa}]$} & {$[\mathrm{Mpa}]$} & {$[\mathrm{Mpa}]$} & {$[\%]$} & {$[\%]$} \\
\hline $\mathrm{X}$ & 0.25 & 0.27 & 0.27 & 7.64 & 8,53 \\
\hline $\mathrm{Y}$ & 0,17 & 0,21 & 0,20 & 17.81 & 13.93 \\
\hline $\mathrm{Z}$ & 2.77 & 3.14 & 3.16 & 13.2 & 13,76 \\
\hline
\end{tabular}

Table 4: stress evaluation at point 3

\begin{tabular}{|c|c|c|c|c|c|}
\hline \multirow{2}{*}{ Direction } & T History & RV Ansys & $\begin{array}{c}\text { RV } \\
(22-23)\end{array}$ & $\begin{array}{c}\text { Error TH } \\
\text { vs. Ansys }\end{array}$ & $\begin{array}{c}\text { Error TH } \\
\text { vs. RV }\end{array}$ \\
\cline { 2 - 6 } & {$[\mathrm{Mpa}]$} & {$[\mathrm{Mpa}]$} & {$[\mathrm{Mpa}]$} & {$[\%]$} & {$[\%]$} \\
\hline $\mathrm{X}$ & 0,23 & 0,26 & 0,26 & 9.65 & 9.32 \\
\hline $\mathrm{Y}$ & 0,03 & 0,26 & 0,03 & - & 11.71 \\
\hline $.3 \mathrm{Z}$ & 2.85 & 3.07 & 3.09 & 7.78 & 8.30 \\
\hline
\end{tabular}

Table 5: stress evaluation at point 4.

\section{ACKNOWLEDGMENT}

Facundo Ignacio Garzon performed the computations here shown in Chapter 3 as a part of his MS thesis. The technical discussions with Prof. Antonietta Lo Conte (Department of Mechanical Engineering - Politecnico di Milano) are also gratefully acknowledged.

\section{REFERENCES}

[1] H. Madsen, Extreme-value statistics for nonlinear stress combination, Journal of Engineering Mechanics 111, 1121-1129, 1985.

[2] D.J. Segalman, G.W.G. Fulcher, G.M. Reese, R.V. Field Jr, An efficient method for calculating R.M.S. Von Mises stress in a random vibration environment, Journal of Sound and Vibration 230(2), 393-410, 2000.

[3] M.B. Priestley, Spectral analysis of non-stationary random processes, Journal of Sound and Vibration 6, 86-97, 1967.

\section{APPENDIX}

$A_{11}=A_{22}=A_{33}=1 ; A_{44}=A_{55}=A_{66}=3 ; A_{12}=A_{13}=A_{23}-\frac{1}{2}$

(other coefficients are zero) 